\title{
Correlations of First and Second Heart Sounds with Age, Sex, and Body Mass Index
}

\author{
Bjarke Skogstad Larsen ${ }^{1}$, Simon Winther ${ }^{2}$, Morten Bøttcher ${ }^{2}$, Louise Nissen ${ }^{2}$, Johannes Struijk ${ }^{1}$, \\ Samuel Emil Schmidt ${ }^{1}$ \\ ${ }^{1}$ Aalborg University, Aalborg, Denmark \\ ${ }^{2}$ Department of Cardiology Hospital Unit West, Herning, Denmark
}

\begin{abstract}
Although auscultation of heart sounds is a commonly used diagnostic tool in preliminary investigation of heart function, no description of normal amplitudes of the two major heart sounds, the first (S1) and second (S2) heart sounds, and their relation to age, sex, and body mass index (BMI), exist.

Heart sound recordings from 739 healthy subjects, from the "Dan-NICAD" study, recorded with Acarix CADScor System at the left fourth inter-costal space (IC4), were investigated. The $S 1$ and $S 2$ sounds were isolated using an automated segmentation algorithm and their absolute amplitudes, $|S 1|$ and $|S 2|$, were tested for correlation with age, sex, and BMI.

Mean $|S 1|$ was $95.4( \pm 6.2) d B$ for men and $93.7( \pm 6.8)$ $d B$ for women, which showed a significant difference $(p<0.05)$. Mean $|S 2|$ was 91.9 ( \pm 5.1$) d B$ for men and 91.8 $( \pm 5.6) d B$ for women, showing no significant difference $(p=0.82) .|S 1|$ and $|S 2|$ were only weakly correlated with age ( $r=-0.084$ and $r=-0.069$ respectively). Both $|S 1|$ and $|S 2|$ were well correlated with BMI $(r=-0.42$ and $r=-0.50$ respectively).

The correlation between BMI and the amplitude of heart sounds is an important baseline knowledge when developing algorithms for automated analysis of heart sounds.
\end{abstract}

\section{Introduction}

As more studies for classification of normal and abnormal heart sounds continue to be published, a baseline investigation of normal heart sounds remains largely unexplored.

Cardiac dimensions are significantly affected by age, sex, height, and weight [1]. This raises the question if some of the characteristic sounds generated by the heart are also correlated with these parameters.

The two major heart sounds heart sounds, S1 and S2, are each produced by the closing of a pair of valves. This study aims to investigate these two heart sounds and their relation with the parameters age, sex and BMI.

Improved knowledge of normal heart sounds and their relation to physiological parameters may also be of use in future related studies.

\section{Methods}

The Dan-NICAD study [2] is a cross-sectional trial that included 1675 patients who were referred to coronary computed tomography angiography (CCTA) due to symptoms suggestive of coronary artery disease (CAD). These patients had no previous history of CAD, or advanced AV-block.

Phonocardiography (PCG) signals were recorded at left IC4 using CADScor System (Acarix A/S, Denmark) in three 8 second intervals from each subject during breath hold.

For the current study, we analysed patients in the DanNICAD study who had no indication of CAD $(n=739)-$ meaning patients who had a coronary artery calcium (CAC) score of 0 and no coronary artery stenosis discovered through CCTA. The subset consisted of $63 \%$ female and the mean age was $54 \pm 8.4(\mathrm{SD})$ years. All further analysis was based on this subset.

\subsection{Signal Analysis}

Heart sound recordings were automatically segmented using a duration dependent hidden Markov model developed by Schmidt et al. [3]. Heart beats were arranged into equal length vectors and aligned to onset of $\mathrm{S} 2$ as seen in Figure 1.

A $4^{\text {th }}$ order Butterworth band-pass filter with cutoff frequencies of $35 \mathrm{~Hz}$ and $800 \mathrm{~Hz}$ was applied to the heart beat signals, and S1 and S2 segments were extracted using the following windows:

S1: $10 \mathrm{~ms}$ prior to detected onset of S1 until $100 \mathrm{~ms}$ prior to detected onset of $\mathbf{S} 2$.

S2: $10 \mathrm{~ms}$ prior to detected onset of S2 until $200 \mathrm{~ms}$ after this onset. 
The maximum absolute amplitude for each of the two segments was identified for each beat, and the median $|\mathrm{S} 1|$ and $|\mathrm{S} 2|$ were calculated for each subject.

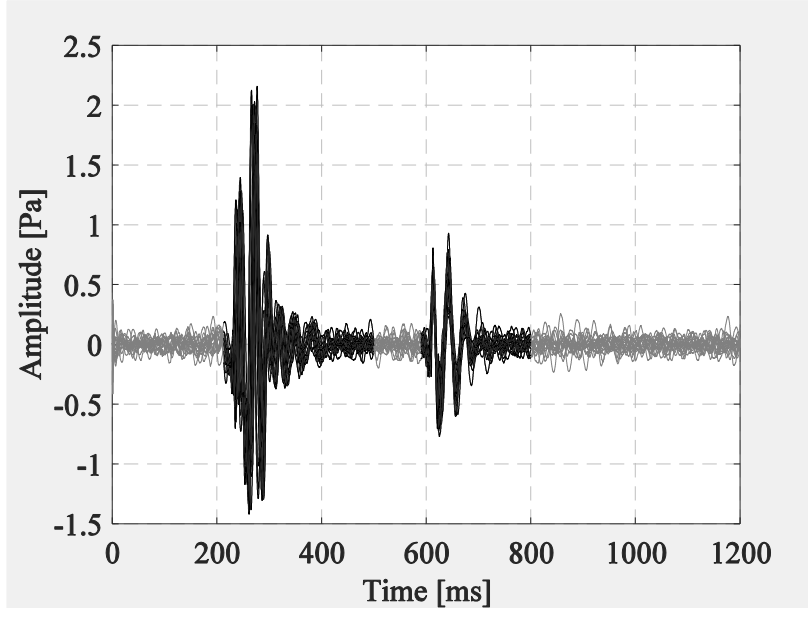

Figure 1. An example of S1 and S2 extraction in multiple heart beats from one subject. The two darker sections are extracted segments of S1 (at around $300 \mathrm{~ms}$ ) and S2 (at around $650 \mathrm{~ms}$ ). Subject: 10001.

\subsection{Statistics}

A two-sample t-test was used to test for equal means of $|\mathrm{S} 1|$ and $|\mathrm{S} 2|$ between sexes.

Pearson's correlation was used to test for correlation of $|\mathrm{S} 1|$ and $|\mathrm{S} 2|$ with Age and BMI.

\section{Results}

Correlations of S1 and S2 amplitudes to sex, age and BMI is illustreted in Table 1. As indicated, there is a significant difference between males and females for $|\mathrm{S} 1|$, while no such difference exists for $|\mathrm{S} 2|$.

Table 1. Correlations of S1 and S2 with age, sex and body mass index.

\begin{tabular}{ccccc} 
& $\begin{array}{c}\text { Male } \\
\text { mean } \\
(\mathbf{s d})\end{array}$ & $\begin{array}{c}\text { Female } \\
\text { mean } \\
(\mathbf{s d})\end{array}$ & $\begin{array}{c}\text { Age } \\
\mathbf{r}\end{array}$ & $\begin{array}{c}\text { BMI } \\
\mathbf{r}\end{array}$ \\
\hline$|\mathrm{S} 1|$ & $\begin{array}{c}95.40 \mathrm{~dB}^{\mathrm{b}} \\
(6.15 \mathrm{~dB})\end{array}$ & $\begin{array}{c}93.68 \mathrm{~dB}^{\mathrm{b}} \\
(6.78 \mathrm{~dB})\end{array}$ & $-0.0843^{\mathrm{a}}$ & $-0.4155^{\mathrm{a}}$ \\
\hline$|\mathrm{S} 2|$ & $\begin{array}{c}91.93 \mathrm{~dB} \\
(5.12 \mathrm{~dB})\end{array}$ & $\begin{array}{l}91.83 \mathrm{~dB} \\
(5.65 \mathrm{~dB})\end{array}$ & -0.0691 & $-0.4974^{\mathrm{a}}$ \\
\hline
\end{tabular}

${ }^{\mathrm{a}}$ significant correlation $(\mathrm{p}<0.05)$

${ }^{b}$ significant difference between sexes $(\mathrm{p}<0.05)$

There was a slight inverse correlation between age and $|\mathrm{S} 1|$ and $|\mathrm{S} 2|$, however only correlation of $|\mathrm{S} 1|$ and age was found to be significant $(\mathrm{p}<0.05)$.
Body mass index was well correlated (inversely) with $|\mathrm{S} 1|$ and $|\mathrm{S} 2|$, and both correlations were found to be significant $(\mathrm{p}<0.05)$.

\section{Discussion}

This study analyzed normal PCG signals for correlation of first and second heart sounds with age, sex and BMI.

While significant difference between males and females was found for $|\mathrm{S} 1|$, this different was small compared to the intra-sex variation of $|\mathrm{S} 1|$. Surprisingly, this difference was nor found for $|\mathrm{S} 2|$.

BMI was inversely correlated with $|\mathrm{S} 1|$ and $|\mathrm{S} 2|$. This correlation is expected as a higher BMI is often associated with a thicker chest wall, and thus a greater attenuation of heart sounds.

\section{Acknowledgements}

We wish to thank Acarix A/S for providing access to the heart sound recordings used in this study.

\section{Conflict of Interest}

Bjarke Skogstad Larsen is an industrial PhD fellow at Acarix A/S and Aalborg University, and is also a minor shareholder in Acarix A/S.

Samuel Emil Schmidt is a minor shareholder in Acarix $\mathrm{A} / \mathrm{S}$, and is also employed at this company as a part-time consultant.

\section{References}

[1] Pfaffenberger S, Bartko P, Graf A, et al. Size Matters! Impact of Age, Sex, Height, and Weight on the Normal Heart Size. Circ Cardiovasc Imaging 2013; 6: 1073 1079.

[2] Nissen L, Winther S, Isaksen C, et al. Danish study of Non-Invasive testing in Coronary Artery Disease (DanNICAD): study protocol for a randomised controlled trial. Trials 2016; 17: 262.

[3] Schmidt SE, Holst-Hansen C, Graff C, et al. Segmentation of heart sound recordings by a durationdependent hidden Markov model. Physiol Meas 2010; 31: 513-529.

Address for correspondence.

Bjarke Skogstad Larsen

Fredrik Bajers Vej 7, C2-203

9220 Aalborg East, Denmark

E-mail: bsl@hst.aau.dk 
Page 3 
Page 4 